\title{
Estratificación de riesgo cardiovascular en enfermedades autoinmunes en un hospital universitario
}

\author{
María Soledad Gálvez Elkin'1, Mariano Montiel Bertone ${ }^{1}$, Cintia Lastra', Isaac Ignacio Cadile ${ }^{1}$, Laura Beatriz Onetti', \\ Eduardo Mussano² \\ ${ }^{1}$ Médicos de planta, ${ }^{2}$ Jefe del Servicio y Director del Posgrado. Servicio de Reumatología, Hospital Nacional de Clínicas. UHMI nº 1 , FCM, UNC.
}

\section{Resumen}

Introducción: Las enfermedades autoinmunes (EAl) han sido consideradas como no fatales; sin embargo, la mayoría de éstas tienen una historia natural de mortalidad prematura. Algunas como el lupus eritematoso sistémico (LES), artritis reumatoidea (AR), esclerosis sistémica (ES), polimiositis, vasculitis y otras, están asociadas a una mortalidad temprana comparable a las enfermedades cardiovasculares y neoplásicas.

Objetivos: 1- Identificar las EAl con mayor riesgo cardiovascular previamente diagnosticadas en pacientes que sean atendidos en un servicio de Reumatología de un hospital universitario. 2- Comparar el riesgo cardiovascular calculado según la escala de Framingham y el modelo SCORE en pacientes con diagnóstico previo de enfermedad autoinmune. Material y métodos: Estudio analítico de corte transversal, en un servicio de Reumatología de un hospital universitario donde se reclutaron 129 historias clínicas de pacientes que acudieron espontáneamente entre el 1 de noviembre de 2010 y el 31 de mayo de 2011. Se elaboraron tablas de cruces de variables y su posterior cálculo con Chi Cuadrado y coeficiente de Pearson.

Resultados: Las EAl con mayor riesgo cardiovascular fueron AR, vasculitis y EASN. La escala de Framingham mostró solo un paciente con AR que tenía RCV muy elevado. La vasculitis encabezó el RCV elevado con el 16,7\%, pero esto corresponde solo a un paciente. El segundo lugar estaba representado por AR (10,7\%), luego EASN (10\%) y LES $(4,5 \%)$. El modelo SCORE demostró que el 3,1\% de las enfermedades tenía muy elevado RCV, porcentaje que estaba comprendido por 3 pacientes con AR y 1 con vasculitis. El RCV elevado estuvo representado sólo por AR en un 13,3\%. Al comparar ambas escalas de riesgo cardiovascular, el 70,5\% de 129 pacientes presentaron bajo RCV. Sólo un paciente $(0,8 \%)$ con bajo RCV en la escala de Framingham tenía muy elevado RCV en el SCORE. De acuerdo al coeficiente de correlación R de Pearson existe un nivel de asociación de casi el $50 \%$, por lo tanto, se demostró que existe muy buena correspondencia entre estas variables. Conclusión: Se encontró una correlación positiva entre las dos escalas, y las enfermedades con mayor riesgo cardiovascular fueron la AR, vasculitis y EASN, destacando el bajo número de casos de las últimas

\section{Summary}

Introduction: Autoimmune diseases (AID) have been considered as non-fatal; however, most of these have a natural history of premature mortality. Some such as lupus erythematosus (SLE), rheumatoid arthritis (RA), systemic sclerosis (SS), polymyositis, vasculitis and others are associated with early mortality comparable to cardiovascular and neoplastic diseases.

Objectives: 1- Identify autoimmune diseases with increased cardiovascular risk in previously diagnosed patients who are treated at a rheumatology service of an university hospital. 2- To compare the cardiovascular risk calculated according to the Framingham and the SCORE model in patients with a previous diagnosis of autoimmune disease treated at a rheumatology service of a public hospital.

Methods: A cross-sectional analytical study in the rheumatology department of an university hospital was carried out where 129 medical records of patients were recruited. They came spontaneously between November 1, 2010 and May 31, 2011. Tables of combination of variables were carried out and its subsequent calculation with the help of the Chi Square statistical coefficient and Pearson coefficient.

Results: The AID with increased cardiovascular risk were RA, vasculitis and EASN. The Framingham showed a single patient with RA who had very high CVR. The high CVR was led by vasculitis with $16.7 \%$, but it must be stressed that this corresponds to a single patient. The second place was represented by RA (10.7\%), then EASN (10\%) and SLE (4.5\%). The SCORE model showed that $3.1 \%$ of the diseases had a very high cardiovascular risk, and this percentage was comprised of 3 patients with RA and 1 with vasculitis. The high CVR was represented only by $13.3 \%$ RA. When comparing the two scales of cardiovascular risk, $70.5 \%$ of 129 patients had low CVR. Only one patient $(0.8 \%)$ with low cardiovascular risk in the Framingham had very high CVR in SCORE. According to the correlation coefficient $R$ of Pearson association exists a level of almost $50 \%$, therefore, it was shown that there is good correspondence between these variables.

Conclusion: We found a positive correlation between the two scales, and diseases with increased cardiovascular risk were RA, vasculitis and

\section{Correspondencia}

Prof. Dr. Eduardo Mussano, Servicio de Reumatología - Hospital Nacional de Clínicas, Santa Rosa 1564, Córdoba.

E-mail: reumahnc@fcm.unc.edu.ar - edemussano@yahoo.com.ar 
dos comparadas con la AR. Notoriamente el nivel de evidencia es mayor en AR y en LES que en cualquier otra patología autoinmune. Esto no descarta que el resto de ellas posea alto RCV. Futuros trabajos multicéntricos deberían plantearse para el desarrollo de guías destinadas al resto de las enfermedades autoinmunes o para la aplicación de las ya existentes.

Palabras clave: riesgo cardiovascular, enfermedades autoinmunes, escala de Framingham, modelo de score.
EASN, highlighting the low number of cases in the last two compared with RA. Notoriously, the evidence level is higher in RA and SLE than in any other autoimmune disease. This does not rule out that the rest of them carry high CVR. Prospective multi-centre studies should consider for the development of guides the rest of autoimmune diseases or for the implementation of existing ones. This work presents no conflict of interest.

Key words: cardiovascular risk, autoimmune diseases, Framingham scale, score model.

atendidos en un servicio de Reumatología de un hospital universitario.

\section{Material y métodos}

Se realizó un estudio analítico de corte transversal donde se revisaron en forma consecutiva todas las historias clínicas de pacientes con diagnóstico previo de enfermedades autoinmunes (EAI) que acudieron espontáneamente por consultorio externo al Servicio de Reumatología del Hospital Nacional de Clínicas (HNC) desde el 01 de noviembre de 2010 al 31 de mayo de 2011. Se seleccionaron los pacientes cuya edad estaba comprendida entre 30 y 74 años, al ser la edad en la que se basan las escalas de riesgo de Framingham ${ }^{2}$.

Muestra: pacientes con diagnóstico previo de enfermedad autoinmune que sean atendidos en el consultorio externo del Servicio de Reumatología del HNC desde 01 de noviembre de 2010 hasta el 31 de mayo de 2011.

\section{Criterios de inclusión:}

1. De ambos sexos, que tengan diagnóstico previo de enfermedades autoinmunes que acuden al consultorio externo del Servicio de Reumatología del HNC.

2. Que tengan más de 30 y menos de 74 años de edad.

3. Que vivan en el país, en diferentes localidades de la provincia de Córdoba o provincias vecinas.

4. Que sean atendidos desde 01 de noviembre de 2010 hasta el 31 de mayo de 2011.

\section{Criterios de exclusión:}

1. Que tengan menos de 30 y más de 74 años de edad.

2. Pacientes extranjeros que no residan en el país.

3. Pacientes que por razones económicas y/u otras razones no regresen a nuevas consultas.

Cabe aclarar que se utilizaron aquellos casos donde estaban completas la totalidad de variables, ya que son un elemento esencial a la hora de calcular el riesgo cardiovascular.

Las variables que se recogieron fueron:

1. Enfermedad autoinmune que padece: se citaron diagnósticos previos de Lupus Eritematoso Sistémico 
(LES), Artritis Reumatoidea (AR), Síndrome de Sjögren Primario (SSp), Esclerosis Sistémica (ES), Síndrome Antifosfolípido Primario (SAFp), Enfermedad Mixta del Tejido Conectivo (EMTC), Vasculitis Sistémica Primaria (VSp) y Dermato/Polimiositis (DM/PM).

2. Edad al momento del diagnóstico: se registró la edad en años cumplidos al momento del diagnóstico de la enfermedad autoinmune.

3. Tiempo de evolución de enfermedad autoinmune: se calculó el tiempo de evolución en años desde la edad de diagnóstico de la enfermedad autoinmune hasta la edad al momento de la recolección de los datos. Se los dividió en:

- Menor a 2 años.

- Entre 3 y 10 años.

- Mayor a 10 años.

4. Tratamiento: se registró si recibían o no al momento de la recolección de datos:

a) corticoides,

b) inmunosupresores / inmunomoduladores / biológicos,

c) antihipertensivos,

d) hipoglucemiantes orales,

e) aspirina.

5. Edad del paciente: se registró la edad en años cumplidos al momento de la recolección de datos.

6. Nivel serológico de colesterol total: se registró el último valor citado en la historia clínica, cuya fecha no debía superar los 60 días previos a la recolección de datos. Si este plazo era mayor, se solicitaba un nuevo control.

7. Nivel serológico de HDL colesterol: se registró el último valor citado en la historia clínica, cuya fecha no debía superar los 60 días previos a la recolección de datos. Si este plazo era mayor, se solicitaba un nuevo control.

8. Medición de tensión arterial (TA): se registró el valor de TA sistólica y diastólica, tomada en la última visita al consultorio externo de Reumatología hasta el momento de recolección de datos.

9. Presencia de Diabetes Mellitus (DBT): se registró a los pacientes que estaban bajo tratamiento con insulina o hipoglucemiantes orales o con diagnóstico previo de DBT al momento de recolección de datos.

10. Tabaquismo: se registró a los pacientes que fumaban cigarrillos regularmente durante los últimos 12 meses a la recolección de datos.

Las escalas para la estimación del riesgo que se utilizaron fueron:

1. Escala de Framingham, versión Wilson 1998: usa edad, sexo, presión arterial sistólica y diastólica, colesterol total, colesterol HDL, diabetes y tabaquismo. Estima el riesgo de evento coronario absoluto ${ }^{2}$.

Se estimó el riesgo utilizando la calculadora http:// www.mdcalc.com/framingham-cardiac-risk-score

2. Escala del proyecto SCORE: usa edad, sexo, presión arterial sistólica, relación colesterol total/HDL colesterol y tabaquismo. Las tablas están elaboradas para población entre 35 y 64 años, por lo que en los pacientes que tenían entre 65 y 74 años la variable de edad fue considerada como si tuvieran 64 años, y los que tenían entre 30 y 34 años, como si tuvieran 35 años ${ }^{6}$. Se utilizó el modelo SCORE para población de alto riesgo, el cual es sugerido en las 10 recomendaciones de manejo del riesgo cardiovascular de EULAR?

Las Guías Europeas de hipertensión proponen una tabla de equivalencias entre riesgo coronario (Framingham) y riesgo de mortalidad cardio y cerebrovascular (SCORE), por lo que se considera bajo riesgo, moderado, elevado y muy elevado con Framingham a $<15 \%, 15-19 \%$, 20-30\% y $>30 \%$, y con SCORE $<4 \%, 4-5 \%, 5-8 \%$ y $>8 \%$, respectivamente $\mathrm{e}^{2,6,7}$.

\section{Técnica de recolección e instrumentos de análisis}

Se reclutaron en forma consecutiva todas las historias clínicas de pacientes con diagnóstico previo de enfermedades autoinmunes que acudieron espontáneamente al consultorio externo del Servicio de Reumatología del HNC desde el 01 de noviembre de 2010 al 31 de mayo de 2011. Para el análisis de los datos, primeramente se estudiaron cada una de las frecuencias de cada una de las variables. Con esto se estimó la distribución de los datos en relación a la variable en estudio. La tabla de frecuencia incluye los distintos valores que presenta la variable acompañados por el número de veces que las clases o categorías seleccionadas aparecen. Estas frecuencias, a su vez, se representan a través de gráficos de sectores para su mejor visualización. Siguiendo con el análisis, se procede a la elaboración de tablas de cruces de variables y su posterior cálculo con la ayuda del coeficiente estadístico Chi Cuadrado. Este coeficiente es muy usual ante la necesidad de hacer una comparación global de grupos de frecuencias. Con este test lo que se quiere determinar es si la frecuencia observada de un fenómeno es significativamente igual a la frecuencia teórica prevista, o si, por el contrario, estas dos frecuencias acusan una diferencia significativa para un nivel de significación por ejemplo del $5 \%$.

\section{Resultados}

De una cohorte de 129 pacientes con diagnóstico previo de enfermedad autoinmune, el 87,6\% (113) eran mujeres y el restante $12,4 \%$ (16) hombres, con una media de edad de 53,17 años. Considerando la medida de tensión arterial sistólica, el 41,9\% tenía menos de $130 \mathrm{mmHg}$, y el 58,1\% (75) tenían HTA, de los cuales sólo 32 recibían antihipertensivos. 15 pacientes (11,6\%) tenían diagnóstico de DBT y dos de ellos $(1,5 \%)$ no estaban tratados. El 89,1\% no fumaron cigarrillos en los últimos 12 meses o nunca lo hicieron. Con respecto al diagnóstico previo de enfermedad autoinmune de los pacientes reclutados, el 58,1\% (75) tenían diagnóstico 
de AR, el 17,1\% (22) LES, 7,8\% (10) EASN, 4,7\% vasculitis, de los que 3 tenían Granulomatosis de Wegener, y el resto, Panarteritis Nodosa (1), Enfermedad de Takayasu (1) y Churg-Strauss (1). 4 pacientes tenían diagnóstico de Esclerosis Sistémica, 3 Síndrome de Sjögren primario y 3 Síndrome de Superposición. Sólo 2 tenían SAF primario y uno EMTC. La media de edad al momento del diagnóstico era entre 40 y 49 años. El 31\% tenían diagnóstico reciente de su enfermedad (menos de 2 años), el 37,2\% (48) más de 10 años de evolución, y el porcentaje restante, una evolución de entre 2 y 10 años. El 46,5\% (60) tenían colesterol total mayor a $200 \mathrm{mg} / \mathrm{dl}$ y el resto, menor a esta cifra. De los 129 pacientes, 68 (52,7\%) recibían corticoterapia, y 104 (80,6\%) tratamiento inmunosupresor. De todos ellos, sólo $18 \mathrm{pa}-$ cientes tomaban aspirina, como antiagregante plaquetario. Utilizando la escala de riesgo de Framingham, el 86,8\% (112) tenían bajo riesgo absoluto de desarrollar enfermedad coronaria en los próximos 10 años, el 3,9\% riesgo moderado, el 8,5\% riesgo elevado y sólo 0,8\% muy elevado. De ellos, sólo un paciente con diagnóstico de AR tenía riesgo muy elevado. La vasculitis era la enfermedad con mayor porcentaje de riesgo elevado (16,7\%), debiendo remarcar que el mismo está representado por un solo paciente. En segundo lugar la AR (10,7\%), seguida de EASN (10\%) y LES (4,5\%). El resto de las EAI tenían RCV bajo.

Al estimar el riesgo con el modelo SCORE se objetivó que el 75,2\% (97) tenían bajo RCV, el 14\% riesgo moderado, el 7,8\% elevado y sólo el 3,1\% muy elevado RCV. De estos últimos, 3 tenían diagnóstico de AR y 1 vasculitis. Sólo pacientes con AR mostraron riesgo elevado, lo que representa un $13,3 \%$. Se mostró riesgo moderado en el 33,3\% de los pacientes con diagnóstico de Síndrome de Sjögren y en el 22,7\% de AR. El bajo RCV se manifestó en el 100\% de los pacientes con LES, ES, EASN, DM/PM, EMTC, Síndrome de Superposición y SAF primario. También tenían bajo RCV el 83,3\% de los pacientes con vasculitis, el 66,7\% con Síndrome de Sjögren y el 60\% con AR. Al comparar ambas escalas de riesgo cardiovascular, el 70,5\% de 129 pacientes presentaron bajo RCV. Sólo un paciente $(0,8 \%)$ con bajo RCV en la escala de Framingham tenía muy elevado RCV en el SCORE; mientras que el coeficiente $\mathrm{R}$ de Pearson mostró un importante nivel de correlación, por lo tanto, se deja ver que existe una buena asociación entre ambos métodos de valoración.

\section{Discusión}

En este estudio se intentó demostrar las patologías autoinmunes con mayor riesgo cardiovascular utilizando las escalas de Framingham y SCORE, así como también, comparar los resultados obtenidos en ambas, ya que, por un lado, es bien conocida la relación que tienen estas enfermedades con ateroesclerosis acelerada y mortalidad prematura de causa cardiovascular, y por el otro, Framingham, que ha sido pionero en el estudio del RCV, presenta básicamente dos problemas:
1- El primero de ellos es que permite una estimación del riesgo coronario, pero no del cardiovascular.

2- El segundo problema es que sobrestima el riesgo coronario en poblaciones de menor riesgo ${ }^{11}$.

Cuando se realizó la búsqueda bibliográfica no se hallaron estudios que comparen estas escalas en EAI.

Existen algunos estudios que intentan valorar concordancias y discrepancias entre estas tablas en pacientes hipertensos, como son el de Maiques y Álvarez, que comparan SCORE y Framingham, Cristóbal y Ramon lo hacen con Framingham calibrada para España o REGICOR y Framingham-Wilson, Baena que compara SCORE y REGICOR con Framingham-Wilson en población general y García Mora compara REGICOR y SCORE en sujetos con algún factor de riesgo cardiovascular ${ }^{12}$.

En el estudio CICLO-RISK donde evalúan las concordancias y discrepancias en la estimación del RCV en pacientes hipertensos con las escalas de FraminghamGrundy, REGICOR y SCORE, encontraron importantes diferencias a la hora de clasificar a los pacientes según niveles de riesgo, especialmente entre esta última comparada con Framingham-Grundy y SCORE. Sus resultados indicaron problemas serios de concordancia entre REGICOR y las otras dos escalas. Sin embargo, entre Framingham y SCORE esto era aceptable.

Este resultado coincidió con el presente estudio, el que incluyó 129 pacientes con diagnóstico de EAI a quienes se les estimó el RCV aplicando las escalas de Framingham y SCORE, y al comparar las mismas se objetivó que el $70,5 \%$ de los pacientes tenían bajo RCV; que las diferencias más marcadas se mostraron en sólo 5 pacientes que tenían RCV bajo en el SCORE y elevado en el Framingham; y que sólo un paciente $(0,8 \%)$ con bajo RCV de la escala de Framingham tenía muy elevado riesgo en el SCORE. De acuerdo al coeficiente de correlación $\mathrm{R}$ de Pearson existe un nivel de correlación de casi el $50 \%$, por lo tanto, se deja ver que existe perfecta asociación entre ambas escalas.

Se sabe bien del incremento del riesgo cardiovascular en pacientes con enfermedades reumáticas como Artritis Inflamatorias y Lupus Eritematoso Sistémico. No se encontró evidencia de estudios multicéntricos en el resto de las EAI, pudiendo esto atribuirse al bajo número de casos que éstas representan.

En este estudio, dentro de las enfermedades con mayor riesgo cardiovascular estaban la AR, vasculitis y EASN. La escala de Framingham mostró a un solo paciente con AR que tenía RCV muy elevado. La vasculitis encabezó el RCV elevado con el $16,7 \%$, pero se debe remarcar que esto corresponde a un paciente solamente. El segundo lugar estaba representado por AR (10,7\%), siguiendo las EASN (10\%) y LES (4,5\%).

En la misma población se utilizó el modelo SCORE, objetivándose que el 3,1\% de las enfermedades tenía muy elevado RCV, porcentaje que estaba comprendido por 3 pacientes con AR y 1 con vasculitis. El RCV elevado estuvo representado sólo por AR en un 13,3\%. Hasta el 2009 
no existían guías para evaluación del riesgo cardiovascular en pacientes con artritis inflamatorias ${ }^{16}$. Recientemente EULAR (European League Against Rheumatism) se basó en la evidencia para el desarrollo de recomendaciones en el manejo del riesgo cardiovascular en Artritis Reumatoidea, Artritis Psoriásica y Espondilitis Anquilosante 7 El score de Framingham parece no haber sido la mejor opción para la evaluación del riesgo de estos pacientes, por no ser lo suficientemente sensible para reflejar el factor de riesgo independiente que representan estas enfermedades reumáticas inflamatorias. Atento a esto, se basaron en el modelo para población de alto riesgo para desarrollar enfermedad cardiovascular fatal en los próximos 10 años de SCORE, el cual es recomendado en las mismas en ausencia de guías locales, y sugiere un factor duplicativo de 1.5 que se aplica sólo a la población con AR y que tiene más de 10 años de evolución de la enfermedad, con positividad para Factor Reumatoideo o anti-CCP, o presencia de manifestaciones extraarticulares ${ }^{7}$. En este estudio, se aplicó este factor al $36 \%$ de la población con $\mathrm{AR}$, por ser seropositivos y tener más de 10 años de evolución de la enfermedad. El principal objetivo de la estimación del RCV es ayudar a la toma de decisiones terapéuticas, tanto en el inicio del tratamiento como en la agresividad de éste. Por esta razón es importante disponer de escalas de riesgo válidas y fiables y que tengan en cuenta las características de la población a la que se aplican. En este momento la más promovida es el SCORE.

En conclusión, se encontró una correlación positiva entre las dos escalas, y las enfermedades con mayor riesgo cardiovascular fueron la AR, vasculitis y EASN, destacando el bajo número de casos de las últimas dos comparadas con la AR. Notoriamente el nivel de evidencia es mayor en $A R$ y en LES que en cualquier otra patología autoinmune. Esto no descarta que el resto de ellas posea alto RCV. Futuros trabajos multicéntricos deberían plantearse para el desarrollo de guías destinadas al resto de las enfermedades autoinmunes o para la aplicación de las ya existentes.

\section{Bibliografía}

1. Alba P. Ateroesclerosis acelerada en enfermedades autoinmunes. Revista Facultad de Ciencias Médicas 2008; 65 (4):107-108.

2. Wilson P, D’Agostino R, Levy D, Belanger A, Silbershatz $\mathrm{H}$. Prediction of coronary heart disease using risk factor categories. Circulation 1998; 97:1837-47.

3. Grundy S, Pasternak R, Greenland P, Smith SJr, Fuster V. Assessment of cardiovascular risk by use of multiple-risk-factor assessment equations: a statement for healthcare professionals from the American Heart Association and the American College of Cardiology. Circulation 1999; 100:1481-92.

4. Aranceta J, Pérez C, Foz M, Mantilla T, Serra L, Moreno B, et al. Grupo Colaborativo para el estudio DORICA fase II. Tablas de evaluación del riesgo coronario adaptadas a la población española. Estudio DORICA Med Clin (Barc). 2004; 123:686-91.

5. Conroy RM, Pyorala K, Fitzgerald AP, Sans S, Menotti A, de Backer G, et al, on behalf of the SCORE Project group. Estimation of ten-year risk of fatal cardiovascular disease in
Europe: the SOCRE Project. Eur Heart J. 2003; 24:987-1003.

6. Ian Grahama, Dan Atara, Knut Borch-Johnsenb, Gudrun Boysend. Guías de práctica clínica sobre prevención de la enfermedad cardiovascular: versión resumida. Rev Esp Cardiol. 2008; 61 (1):e1-e49.

7. Peters MJL, Symmons DPM, McCarey D, et al. EULAR evidence-based recommendations for cardiovascular risk management in patients with rheumatoid arthritis and other forms of inflammatory arthritis. Ann Rheum Dis. 2010; 69:325-331.

8. Jeffrey S. Berger, MS, Courtney O. Jordan, MD, Donald Lloyd-Jones, MD. Screening for cardiovascular risk in asymptomatic patients. Journal of the American College of Cardiology Vol. 55, No. 12, 2010.

9. Jaume Marrugata, Pascual Solanas, Ralph D’Agostinod, Lisa Sullivand. Estimación del riesgo coronario en España mediante la ecuación de Framingham calibrada. Rev Esp Cardiol. 2003; 56 (3):253-61.

10. Paulino González-Diego, Conchi Moreno-Iribas, María Jesús Guembea. Adaptación de la función de riesgo coronario de Framingham-Wilson para la población de Navarra (RICORNA). Rev Esp Cardiol. 2009; 62 (8):875-85.

11. Brotons C, Cascant P, Ribera A, Moral I, Permanyer G. Utilidad de la medición del riesgo coronario a partir de casos y controles. Med Clin (Barc). 2003; 121:327-30.

12. García-Ortiz L, Gómez-Marcos MA, GonzálezElena LJ, Rodríguez-Sánchez E, García García Á. Framingham-Grundy, REGICOR y SCORE en la estimación del riesgo cardiovascular del paciente hipertenso. Concordancias y discrepancias (CICLO-RISK). Hipertensión (Madr). 2006; 23 (4):111-7.

13. Avouac J, Allanore Y. Cardiovascular risk in rheumatoid arthritis: effects of anti-TNF drugs. Expert Opin Pharmacother. 2008; 9:1121-1128.

14. Stephanie O. Keeling \& Michelle Teo \& Daisy Fung. Lack of cardiovascular risk assessment in inflammatory arthritis and systemic lupus ery thematosus patients at a tertiary care center. Clin Rheymatol DOI 10.1007 / s10067-011-1747-9.

15. Michael T Nurmohamed, George Kitas. Cardiovascular risk in rheumatoid arthritis and diabetes: how does it compare and when does it start? Ann Rheum Dis. June 2011. Vol 70 No 6.

16. Murray B. Urowitz, MD; Dafna D. Gladman, MD. The SLICC Inception Cohort for Atherosclerosis. Current Rheumatology Reports. 2008; 10:281-285.

17. Meune C, Touzé E, Trinquart L, Allanore Y. Trends in cardiovascular mortality in patients with rheumatoid arthritis over 50 years: a systematic review and meta-analysis of cohort studies. Rheumatol (Oxford) 2009; 48:1309-1313.

18. Rasker JJ and Cosh JA. Cause and age at death in a prospective study of 100 patients with rheumatoid arthritis. Annals of the Rheumatic Diseases 1981, 40:115-120.

19. Guillermo J. Pons-Estel, Luis A. Gonzalez, Jie Zhang. Predictors of cardiovascular damage in patients with systemic lupus erythematosus: data from LUMINA (LXVIII), a multiethnic US cohort. Rheumatology 2009; 48:817-822.

20. Young Hee Rho, MD, PhD, Cecilia P. Chung, MD. Inflammatory mediators and premature coronary atherosclerosis in rheumatoid arthritis. Arthritis Rheum. 2009. November 15; 61 (11):1580-1585. 